\title{
Editorial: Journal of Classification Vol. 38-3
}

\author{
Paul D. McNicholas ${ }^{1}$
}

Accepted: 10 November 2021

(C) The Author(s) under exclusive licence to The Classification Society 2021

In the first of the ten articles herein, Chen, Sun, Euan and Ombao introduce two algorithms for clustering data on brain activity; specifically, electroencephalograms. The second article, by Michis, develops a wavelet multidimensional scaling approach and applies it to data on economic sentiment. In the third paper, Ballante, Galvani, Uberti and Figini introduce polarized classification trees and carry out a simulation study to compare their method to established approaches. The fourth paper, by Köhn and Chiu, lays out the completeness conditions of unstructured Q-matrices for the DINA model. In the fifth paper, de Raadt, Warrens, Bosker and Kiers compare reliability coefficients - including kappa coefficients and correlation coefficients - for ordinal rating scales.

In the sixth paper of this issue, Ke and Chang propose a subject selection method for non-parametric model-based classifiers. In the seventh paper, Tomarchio, McNicholas and Punzo extend cluster-weighted models (CWMs; Gershenfeld, 1997) to the matrix variate case. The next article, by $\mathrm{Lu}, \mathrm{Li}$ and Love, considers the parsimonious Gaussian mixture model family (McNicholas \& Murphy, 2008) and introduces a reversible-jump Markov chain Monte Carlo procedure for parameter estimation. The ninth paper of this issue is the second to consider CWMs. Specifically, Soffritti develops three approaches for estimating the covariance matrix of the maximum likelihood estimator under a linear CWM. In the tenth and final paper of this issue, Casa, Bouveyron, Erosheva and Menardi consider coclustering of time-dependent data. Specifically, they propose a co-clustering approach for grouping individuals and variables simultaneously that can handle both functional and longitudinal data. In a particularly timely application, they illustrate their approach on data describing the evolution of the COVID-19 pandemic across 38 countries.

Paul D. McNicholas

mcnicholas@math.mcmaster.ca

1 Department of Mathematics \& Statistics, McMaster University, Hamilton, ON, Canada 


\section{References}

Gershenfeld, N. (1997). Nonlinear inference and cluster-weighted modeling. Annals of the New York Academy of Sciences, 808(1), 18-24.

McNicholas, P. D., \& Murphy, T. B. (2008). Parsimonious Gaussian mixture models. Statistics and Computing, 18(3), 285-296.

Publisher's Note Springer Nature remains neutral with regard to jurisdictional claims in published maps and institutional affiliations. 